\title{
Personality Features and Personality Disorders in Chronic Fatigue Syndrome: A Population-Based Study
}

\author{
Urs M. Nater ${ }^{a, b} \quad$ James F. Jones ${ }^{a} \quad$ Jin-Mann S. Lin ${ }^{a} \quad$ Elizabeth Maloney ${ }^{a}$ \\ William C. Reeves ${ }^{a}$ Christine Heim ${ }^{b}$ \\ ${ }^{a}$ Chronic Viral Diseases Branch, Coordinating Center for Infectious Diseases, Centers for Disease Control and \\ Prevention, and ${ }^{b}$ Department of Psychiatry and Behavioral Sciences, Emory University School of Medicine, \\ Atlanta, Ga., USA
}

\section{Key Words}

Chronic fatigue syndrome $\cdot$ Personality $\cdot$ Personality

disorders · Population-based study

\begin{abstract}
Background: Chronic fatigue syndrome (CFS) presents unique diagnostic and management challenges. Personality may be a risk factor for CFS and may contribute to the maintenance of the illness. Methods: 501 study participants were identified from the general population of Georgia: 113 people with CFS, 264 with unexplained unwellness but not CFS (insufficient fatigue, ISF) and 124 well controls. We used the Personality Diagnostic Questionnaire, 4th edition, to evaluate DSM-IV personality disorders. We used the NEO FiveFactor Inventory to assess personality features (neuroticism, extraversion, openness, agreeableness and conscientiousness). The Multidimensional Fatigue Inventory measured 5 dimensions of fatigue, and the Medical Outcomes Survey Short Form 36 measured 8 dimensions of functional impairment. Results: Twenty-nine percent of the CFS cases had at least 1 personality disorder, compared to $28 \%$ of the ISF cases and $7 \%$ of the well controls. The prevalence of paranoid, schizoid, avoidant, obsessive-compulsive and depressive personality disorders were significantly higher in CFS and
\end{abstract}

ISF compared to the well controls. The CFS cases had significantly higher scores on neuroticism, and significantly lower scores on extraversion than those with ISF or the well controls. Personality features were correlated with selected composite characteristics of fatigue. Conclusions: Our results suggest that CFS is associated with an increased prevalence of maladaptive personality features and personality disorders. This might be associated with being noncompliant with treatment suggestions, displaying unhealthy behavioral strategies and lacking a stable social environment. Since maladaptive personality is not specific to CFS, it might be associated with illness per se rather than with a specific condition.

Copyright $\odot 2010$ S. Karger AG, Basel

\section{Introduction}

Chronic fatigue syndrome (CFS) is an important public health problem with unique diagnostic and management challenges. Studies using population-based ran-

Part of these analyses have been presented at the 67th Annual Meeting of the American Psychosomatic Society.

\section{KARGER}

๑ 2010 S. Karger AG, Basel

Fax +41613061234 E-Mail karger@karger.ch www.karger.com
William C. Reeves, MD

Centers for Disease Control and Prevention

Mail Stop A-15

Atlanta, GA 30333 (USA)

Tel. +1 404639 0221, Fax +1 404639 2779, E-Mail wcr1@ @dc.gov 
dom samples have estimated that CFS prevalence among adults ranges from 0.2 to $2.5 \%$ [1-3]. Most of the people affected have been ill for 5 years or longer, and a quarter are unemployed or receive disability benefits [4]. In spite of this morbidity, only $50 \%$ of those suffering from the illness have consulted a physician [5]. We have recently examined qualitative types of healthcare utilization barriers and found 7 reoccurring barriers: attitudes, self-diagnosis, finances, time constraints, healthcare coverage, fear, and lack of trust and confidence in health professionals [6]. It might be suggested that these barriers are influenced by differential aspects of a person's character, thoughts and feelings, i.e. his or her personality. A better understanding of how personality is related to CFS might improve our understanding of this illness and the care seeking behavior of individuals with the syndrome.

The role of maladaptive personality as a risk factor for developing CFS, as a factor contributing to its morbidity, pursuit of and responsiveness to treatment, or as a consequence of this chronic complex illness has been poorly explored. Published studies have reported around $40 \%$ of persons with CFS as having personality disorders, a relatively high occurrence [7-10]. These studies considered personality on a categorical basis.

However, it may be more productive to consider personality as a dimensional construct, with disorders reflecting extremes on a wide spectrum. The 5 -factor model provides a structure of normal personality traits. These include: (1) neuroticism - the tendency to experience negative affect and affective instability; (2) extraversion - a disposition toward energetic activity, sociability and positive affect; (3) openness to experience - an interest in novel people, ideas and things, as well as intellectual and aesthetic tendencies; (4) agreeableness - the tendency toward warmth, amiability and trust; and (5) conscientiousness - qualities such as diligence, goal orientation, fastidiousness and dependability [11]. Previous studies have found higher scores of maladaptive personality traits in CFS patients compared to healthy controls [12]. In particular, studies have examined the relationship between fatigue and neuroticism, which may also be characterized by a tendency to display high emotional reactivity. Neuroticism has also been associated with negative health outcomes [13]. In general, a positive association between neuroticism and fatigue has been found [14], with CFS patients showing higher levels compared to healthy controls [15] and the general population [16]. These studies clearly highlight the important role of neuroticism in CFS. Other studies which have also examined other personality traits have yielded inconclusive results [12].

Personality Features and Disorders in Chronic Fatigue Syndrome
Published studies evaluating personality associated with CFS have 2 major limitations. First, they enrolled CFS patients from primary or tertiary care centers. The potential effect of self-selection into medical care settings by higher (or lower) scores in neuroticism or any other dimension of personality could not be evaluated. Equally important, as noted in a recent review, associations between neuroticism and CFS likely reflect methodological issues such as differences in assessment instruments, patient selection criteria and CFS case definition, or the absence of an appropriate control group [12]. In the current study, we used standardized validated instruments to (1) describe the prevalence of personality disorders in a population-based sample of persons with and without fatigue, (2) describe personality dimensions in persons with and without fatigue, and (3) relate dimensions of personality in CFS to illness-specific symptoms. We also used standardized and validated measures to define CFS, other unexplained fatiguing illness not meeting the criteria for CFS (we term this 'insufficient number of symptoms' or 'insufficient fatigue severity', ISF), and well controls.

\section{Methods}

This study adhered to US Department of Health and Human Services human experimentation guidelines and received institutional review board approval from the CDC (Centers for Disease Control and Prevention) and collaborating institutions. After a complete description of the study, all participants gave their written informed consent.

\section{Study Design}

Study participants were identified during a survey of unwellness in metropolitan, urban and rural populations of Georgia, conducted between September 2004 and July 2005 [17]. In brief, the study used random digit dialing to screen approximately 3,000 metropolitan households, about 4,000 urban residences and about 5,000 residences in rural areas. Detailed telephone interviews were completed on 5,623 people aged 18-59 years, who were identified by a household informant as 'well', 'unwell without fatigue' and 'unwell with fatigue', representing response rates of 56, 67 and $71 \%$, respectively. Based on their responses in the detailed telephone interviews, the subjects entering the 1-day clinical evaluation had been classified as (1) CFS-like, (2) chronically unwell, and (3) well. Of those who fit the criteria for the CFS-like group, 469 volunteers were eligible for clinical evaluation based on a detailed telephone interview, and 292 (62\%) completed the clinical assessment. Of the eligible well, 481 were invited to participate, based on having matched the CFS-like subjects for age ( \pm 3 years), sex, race/ethnicity and geographic stratum, and 223 (46\%) completed the clinical assessment. Of those with chronic unwellness (having at least 1 of the 4 most common CFS-defining symptoms - fatigue, cognitive impairment, unrefreshing sleep, and 
muscle or joint pain - for $\geq 6$ months), 505 people were randomly selected to participate, and 268 (53\%) completed the clinical assessment.

\section{Clinical Assessment}

During the clinical evaluation, we identified medical and psychiatric exclusionary and comorbid conditions and classified the participants as CFS, ISF or well. A review committee of CDC and Emory University physicians and psychologists reviewed the medical history and clinical, laboratory and psychiatric evaluations to determine the presence of medical and psychiatric conditions.

\section{Psychiatric and Medical Conditions}

To identify exclusionary psychiatric conditions as stipulated by the case definition $[18,19]$, licensed and specifically trained psychiatric interviewers administered a semistructured interview. To screen for medical conditions considered exclusionary for CFS, the participants completed past medical history questionnaires. A standardized physical examination was performed. Blood and urine specimens were obtained for laboratory screening tests to identify possible underlying or contributing medical conditions $[18,19]$.

\section{Measurement of Personality}

Personality Diagnostic Questionnaire, 4th edition. The Personality Diagnostic Questionnaire, 4th edition (PDQ-4) [20], is a 100 -item, self-administered, true/false questionnaire that yields personality diagnoses consistent with the DSM-IV diagnostic criteria for axis II disorders. In the current study, the participants completed the instrument on a computer during their clinical evaluation. The PDQ-4 assesses the 10 personality disorders of the DSM-IV, and 2 additional personality disorders described in the appendix B of the DSM-IV [21]. The PDQ total score provides an index of overall personality disturbance and is calculated by summing up all the pathological responses. A total score of $\geq 30$ indicates that the respondent likely has a personality disturbance. The PDQ-4 was designed for high sensitivity at the expense of low specificity.

NEO Five-Factor Inventory. The NEO Five-Factor Inventory (NEO-FFI) [11] is a 60-item short form of the NEO Personality Inventory. The NEO-FFI assesses 5 personality domains: neuroticism, extraversion, openness to experience, agreeableness and conscientiousness. The reliability coefficients (Cronbach's $\alpha$ ) that we found in our study for the 5 scales were the following: 0.89 (neuroticism), 0.82 (extraversion), 0.72 (openness), 0.74 (agreeableness) and 0.82 (conscientiousness).

\section{Classification of Subjects}

CFS is defined by (1) clinically unexplained (i.e. by other primary diagnoses that might explain fatigue), persistent or relapsing fatigue of at least 6 months' duration that is not the result of ongoing exertion, is not substantially alleviated by rest and results in a substantial reduction in previous levels of occupational, educational, social or personal activities, and (2) a concurrent occurrence of at least 4 accompanying symptoms (unusual postexertional malaise, unrefreshing sleep, significant impairment of memory/concentration, headache, muscle pain, joint pain, sore throat and tender lymph nodes) [18]. As recommended by the international CFS study group [19], the study classified the partici- pants as CFS, ISF (i.e. not meeting the criteria for CFS [18]) or well, using standardized instruments to measure symptoms and functioning (i.e. the Multidimensional Fatigue Inventory to assess fatigue status, the Medical Outcomes Survey Short Form 36 to measure functional impairment, and the CDC Symptom Inventory to evaluate the occurrence, frequency and severity of the 8 CFS-defining symptoms). Cutoffs were used as in previous CDC population-based studies of CFS [1].

\section{Statistical Analyses}

We used the Kolmogorov-Smirnov test to evaluate whether data were normally distributed. For metric data, group differences between CFS, ISF and well were calculated by general linear models. Homogeneity of variance was assessed using Levene's test. For nonmetric data, differences between the 3 groups were analyzed using the Kruskal-Wallis analysis of variance. For multiple comparisons of means, Bonferroni-corrected results were used. Correlations between scores were computed using Spearman's rho correlations. The analyses were two-tailed, with the level of significance set at $\mathrm{p}<0.05$.

\section{Results}

Age, sex and race distributions were similar among the participants with CFS (mean age 44.3 years; $81.4 \%$ female; $74.3 \%$ white) and ISF (mean age 43.1 years; $76.1 \%$ female; $74.2 \%$ white), and the well controls (mean age 44.5 years; $75.0 \%$ female; $76.6 \%$ white) (all comparisons were nonsignificant).

At least 1 personality disorder was diagnosed in 33/113 (29.2\%) persons with CFS, 74/264 (28\%) persons with ISF, and $9 / 124(7.3 \%)$ well persons. The prevalence rates were significantly higher in the CFS and ISF groups compared to the well (table 1). This distribution was also reflected in the PDQ summary score, which was significantly different between the 3 groups, with the highest scores in the CFS group and the lowest in the well group (CFS: 23.29; ISF: 17.7; well: 9.3; $\mathrm{F}(2,494)=51.28 ; \mathrm{p}<0.001$; post hoc test: all comparisons $\mathrm{p}<0.001)$.

The 3 groups differed significantly in their mean scores for several of the $5 \mathrm{NEO}$-FFI scales. The CFS group had the highest mean score for neuroticism, while the well group had the lowest mean score for neuroticism (table 2). Those with CFS had the lowest mean extraversion scores, and the well group had the highest mean scores. Well participants had significantly higher mean agreeableness and conscientiousness scores than the CFS or ISF groups, whereas the scores of the latter 2 groups were similar. The openness scale scores were similar for the 3 diagnostic groups.

Further, we were interested in whether the presence or absence of a personality disorder diagnosis resulted in 
Table 1. PDQ-4 personality disorder diagnoses in cases with CFS and ISF and in well controls

\begin{tabular}{lllllr}
\hline & CFS & ISF & Well & Statistics & p \\
\hline Paranoid PD & $6 / 110(5.5 \%)$ & $13 / 263(4.9 \%)$ & $3 / 124(2.4 \%)$ & $\chi^{2}(4)=6.3$ & 0.175 \\
Schizoid PD & $7 / 110(6.4 \%)$ & $9 / 263(3.4 \%)$ & $2 / 124(1.6 \%)$ & $\chi^{2}(4)=20.2$ & $<0.001$ \\
Schizotypal PD & $3 / 110(2.7 \%)$ & $5 / 263(1.9 \%)$ & $1 / 124(0.8 \%)$ & $\chi^{2}(4)=12.5$ & 0.014 \\
Histrionic PD & $3 / 110(2.7 \%)$ & $3 / 263(1.1 \%)$ & 0 & $\chi^{2}(4)=12.1$ & 0.017 \\
Narcissistic PD & $4 / 110(3.6 \%)$ & $3 / 263(1.1 \%)$ & 0 & $\chi^{2}(4)=5.9$ & 0.203 \\
Borderline PD & $2 / 110(1.8 \%)$ & $1 / 263(0.4 \%)$ & 0 & $\chi^{2}(4)=22.7$ & $<0.001$ \\
Antisocial PD & 0 & 0 & N/A & N/A \\
Avoidant PD & $6 / 110(5.5 \%)$ & $13 / 263(4.9 \%)$ & $2 / 124(1.6 \%)$ & $\chi^{2}(4)=20.2$ & $<0.001$ \\
Dependent PD & 0 & 0 & N/A & N/A \\
Obsessive-compulsive PD & $16 / 110(14.5 \%)$ & $34 / 263(12.9 \%)$ & $4 / 124(3.2 \%)$ & $\chi^{2}(4)=41.2$ & $<0.001$ \\
Negativistic PD & $3 / 110(2.7 \%)$ & $12 / 263(4.6 \%)$ & 0 & $\chi^{2}(4)=12.5$ & 0.014 \\
Depressive PD & $7 / 110(6.4 \%)$ & $15 / 263(5.7 \%)$ & 0 & $\chi^{2}(4)=38.3$ & $<0.001$ \\
\hline
\end{tabular}

$\mathrm{PD}=$ Personality disorder.

Table 2. Distribution of summary NEO-FFI scores for personality dimensions in cases with CFS and ISF and in well controls

\begin{tabular}{llllll}
\hline & CFS $(\mathrm{n}=113)$ & ISF $(\mathrm{n}=264)$ & Well $(\mathrm{n}=124)$ & d.f. & $\mathrm{p}$ \\
\hline Neuroticism & $1.98(1.84-2.12)$ & $1.67(1.58-1.75)$ & $0.92(0.83-1.01)$ & $\mathrm{F}(2,495)=84.01$ & $<0.001^{\mathrm{a}-\mathrm{c}}$ \\
Extraversion & $2.04(1.93-2.15)$ & $2.24(2.18-2.31)$ & $2.66(2.57-2.75)$ & $\mathrm{F}(2,497)=41.34$ & $<0.001^{\mathrm{b}-\mathrm{d}}$ \\
Openness & $2.27(2.19-2.35)$ & $2.19(2.13-2.26)$ & $2.29(2.20-2.38)$ & $\mathrm{F}(2,496)=1.87$ & 0.155 \\
Agreeableness & $2.79(2.71-2.88)$ & $2.79(2.74-2.84)$ & $3.06(3.00-3.13)$ & $\mathrm{F}(2,497)=17.01$ & $<0.001^{\mathrm{b}, \mathrm{c}}$ \\
Conscientiousness & $2.71(2.61-2.81)$ & $2.82(2.76-2.88)$ & $3.07(2.99-3.15)$ & $\mathrm{F}(2,497)=16.56$ & $<0.001^{\mathrm{b}, \mathrm{c}}$ \\
\hline
\end{tabular}

In the CFS, ISF and well columns, values in parentheses denote $95 \%$ CI. Post hoc tests, Bonferroni-corrected. ${ }^{a}$ CFS versus ISF: $\mathrm{p}<$ $0.001 ;{ }^{b}$ CFS versus well: $\mathrm{p}<0.001 ;{ }^{\mathrm{c}}$ ISF versus well: $\mathrm{p}<0.001 ;{ }^{\mathrm{d}}$ CFS versus ISF: $\mathrm{p}<0.01$.

differential personality traits as measured by the NEOFFI. As table 3 indicates, those with at least 1 personality disorder differed from those without personality disorders with respect to mean scores for neuroticism and agreeableness scales, and there was a tendency toward significant differences for the other dimensions.

Finally, personality dimensions were associated with dimensions of fatigue (measured by the Multidimensional Fatigue Inventory) and functional impairment (measured by the Medical Outcomes Survey Short Form 36). Among the 5 personality dimensions, the strongest correlations were found between neuroticism and both functional impairment and fatigue in CFS and ISF. Correlations of these factors with extraversion were similarly high, but the directions of the association were inverted (data not shown; the authors are happy to provide a full table of correlation coefficients upon request).

Personality Features and Disorders in Chronic Fatigue Syndrome

\section{Discussion}

Categorically, $29 \%$ of our participants with CFS had a personality disorder, compared to $7 \%$ of the well controls. Our findings are reminiscent of previous studies which showed that almost $40 \%$ of CFS patients had at least 1 personality disorder $[7,8,10]$. Interestingly, both our study and 2 of the earlier studies found obsessive-compulsive personality disorder to be the most common personality disorder $[7,8]$. One of the key features of obsessive-compulsive personality disorder is perfectionism, which has been shown to be markedly increased in patients with CFS [22]. Another study found that histrionic (23\%) and borderline (17\%) personality disorders were most common in CFS patients [10], and a study of chronic fatigue showed that dependent (11\%), histrionic (13\%) and obsessive-compulsive (16\%) personality disorders

Psychother Psychosom 2010;79:312-318 
Table 3. NEO-FFI dimensions in subjects with and without at least one personality disorder diagnosis in cases with CFS and ISF and in well controls

\begin{tabular}{|c|c|c|c|c|c|}
\hline Prevalence of $\geq 1 \mathrm{PD}$ & $\begin{array}{l}\text { CFS mean } \\
(33 / 113 ; 29.2 \%)\end{array}$ & $\begin{array}{l}\text { ISF mean } \\
(74 / 264 ; 28 \%)\end{array}$ & $\begin{array}{l}\text { Well mean } \\
(9 / 124 ; 7.3 \%)\end{array}$ & d.f. & $\mathrm{p}$ \\
\hline $\mathrm{N}$ with $\mathrm{PD}$ & $2.27(2.03-2.52)$ & $1.95(1.80-2.11)$ & $1.04(0.85-1.23)$ & $\mathrm{F}(2,113)=12.14$ & $<0.001^{\mathrm{a}, \mathrm{b}}$ \\
\hline $\mathrm{N}$ without $\mathrm{PD}$ & $1.86(1.70-2.02)^{* *}$ & $1.55(1.46-1.65)^{* * *}$ & $0.91(0.82-1.01)$ & $\mathrm{F}(2,379)=59.83$ & $<0.001^{\mathrm{a}-\mathrm{c}}$ \\
\hline E with PD & $1.94(1.76-2.12)$ & $2.13(2.00-2.25)$ & $2.30(1.80-2.80)$ & $\mathrm{F}(2,113)=2.04$ & 0.135 \\
\hline E without PD & $2.08(1.95-2.22)$ & $2.29(2.21-2.37)^{*}$ & $2.69(2.60-2.78)^{*}$ & $\mathrm{~F}(2,381)=33.66$ & $<0.001^{\mathrm{a}-\mathrm{c}}$ \\
\hline O with PD & $2.18(2.03-2.33)$ & $2.16(2.04-2.28)$ & $2.02(1.65-2.39)$ & $\mathrm{F}(2,112)=0.4$ & 0.668 \\
\hline $\mathrm{O}$ without $\mathrm{PD}$ & $2.31(2.21-2.41)$ & $2.20(2.13-2.28)$ & $2.31(2.21-2.40)$ & $\mathrm{F}(2,381)=1.92$ & 0.148 \\
\hline A with PD & $2.60(2.41-2.80)$ & $2.62(2.51-2.72)$ & $2.79(2.38-3.21)$ & $\mathrm{F}(2,113)=0.56$ & 0.573 \\
\hline A without PD & $2.87(2.78-3.00)^{* *}$ & $2.86(2.80-2.92)^{* * *}$ & $3.08(3.01-3.15)^{*}$ & $\mathrm{~F}(2,381)=11.36$ & $<0.001^{\text {b,d }}$ \\
\hline C with PD & $2.65(2.45-2.84)$ & $2.67(2.53-2.81)$ & $3.07(2.67-3.47)$ & $\mathrm{F}(2,113)=2.16$ & 0.120 \\
\hline C without PD & $2.74(2.61-2.86)$ & $2.88(2.81-2.95)^{* *}$ & $3.07(2.99-3.15)$ & $\mathrm{F}(2,381)=12.12$ & $<0.001^{\mathrm{a}, \mathrm{e}}$ \\
\hline
\end{tabular}

In the CFS, ISF and well columns, values in parentheses denote 95\% CI. Post hoc tests, Bonferroni-corrected. PD = Personality disorder; $\mathrm{N}=$ neuroticism; $\mathrm{E}=$ extraversion; $\mathrm{O}=$ openness; $\mathrm{A}=$ agreeableness; $\mathrm{C}=$ conscientiousness. ${ }^{\mathrm{a}} \mathrm{CFS}$ versus well: $\mathrm{p}<0.001$; ${ }^{\mathrm{b}}$ ISF versus well: $\mathrm{p}<0.001$; ${ }^{\mathrm{c}}$ CFS versus ISF: $\mathrm{p}<0.01$; ${ }^{\mathrm{d}}$ CFS versus well: $\mathrm{p}<0.01$; ${ }^{\mathrm{e}}$ ISF versus well: $\mathrm{p}<0.01$. ${ }^{*} \mathrm{p}<0.05,{ }^{* *} \mathrm{p}<0.01$, ${ }^{* * *} \mathrm{p}<0.001$; independent $\mathrm{t}$ tests.

were most common [9]. Interestingly, the occurrence of personality disorders in our study was substantially lower than in these other studies. We believe this is best explained by the fact that previously published studies enrolled participants from primary or tertiary care centers, whereas our study was population-based. Patients recruited from primary or tertiary care centers may have more comorbid conditions than subjects recruited from the community.

Examining the dimensionality of personality features, we found increased neuroticism scores in CFS compared to the control group. Neuroticism is defined as a predisposition to experience negative affect, i.e. anxiety and depression. Persons with higher scores in neuroticism are more likely to be noncompliant with treatment suggestions, display unhealthy behavioral strategies, lack a stable social environment and are therefore prone to illness. Neuroticism may also be characterized as a proneness to experience stress. It is interesting to see our results in the context of recent findings showing that high levels of perceived stress are associated with the manifestation of CFS $[23,24]$. As stressful experiences seem to play an important role in triggering CFS symptoms, it might be argued that increased levels of neuroticism might mediate this relationship. Further, we found decreased extraversion scores in CFS. Energetic activity and sociability, the 2 key features of extraversion, may explain this finding. Clearly, CFS is characterized by low levels of energy, and social interactions are down to a minimum. Also, decreased agreeableness and conscientiousness scores were found in CFS. Both personality traits might affect compliance with treatment regimes. Finally, no differences were found for openness.

Our study differs from other studies in that it also included a group of chronically fatigued participants who did not fulfill the criteria for a CFS diagnosis because they had an insufficient number of symptoms or insufficient fatigue severity (this group was termed ISF). Persons with ISF were very similar to those with CFS with respect to personality disorders and certain personality traits although there were a few notable differences. However, current categorical approaches to the diagnosis of CFS might be complemented by a measurement of dimensional features in specific variables of interest. While subjects with ISF fared better than CFS cases with regard to their clinical features, the overall level of illness burden was significantly higher than in well persons. In this context, it is relevant to note that studies comparing CFS to other chronic illnesses, such as multiple sclerosis, did not find differences regarding the prevalence of comorbid personality disorders $[9,10]$. Personality disorders are therefore unlikely to be specific to CFS, but might be related to the chronicity and severity of an illness. Thus, our findings have an impact beyond the field of CFS research and should be considered in the context of chronic illness in general.

The question arises whether personality dispositions are a premorbid risk factor, or whether they are a conse- 
quence of the chronicity and severity of the illness known as CFS (and comorbid conditions), or both. While some authors have raised the possibility that personality alterations are merely sequelae of the illness itself [25], a recent study in a population-based sample of twins found that emotional instability assessed 25 years earlier was predictive of a later diagnosis of chronic fatigue [23]. Although that study did not evaluate CFS, its finding is still relevant for the understanding of the relationship between personality and CFS because its use of a prospective, longitudinal design allows strong inferences to be made regarding the potentially causative contribution of personality to the development of fatiguing illness. In contrast, a recent analysis using data from the British birth cohort study found that personality factors such as neuroticism and extraversion did not predict a diagnosis of CFS [26]. It needs to be noted, though, that this study relied on selfreported diagnoses of CFS. Finally, the question remains whether the severity of the illness might influence trait characteristics such as neuroticism. Feeling unwell might have an impact on how questions regarding personality and other psychological constructs are answered $[27,28]$. Our design did not allow controlling this possibility. We also measured depression and found that neuroticism was higher, and extraversion, openness, agreeableness and conscientiousness were lower in subjects with high depression scores (results not shown). The question of whether personality is primarily a predisposing factor for or a result of full-blown diagnostic CFS therefore still awaits empiric evaluation.

Although we found that personality disorders and maladaptive personality features were relatively common in persons with CFS, more than $70 \%$ of our CFS sample did not fulfill the diagnosis for a personality disorder. Clearly, persons suffering from a comorbid personality disorder might need special attention when being treated. For example, cognitive behavioral therapy and graded exercise therapy are widely held to be the most effective treatments for CFS although their results are inconsistent [29]. Such therapies require that patients understand, adhere to and practice specific activities to manage their thoughts and expenditure of physical energy. The maladaptive personality features that we describe interfere with the ability to follow directions and maintain the selfmotivation needed for cognitive behavioral therapy and graded exercise therapy to be effective. Our findings suggest that a successful treatment of persons with CFS may require special attention to address maladaptive personality therapeutically. Further, maladaptive personality features might further the risk of negatively affecting the doctor-patient relationship by their interference with the patients' motivation to attain by self-care a long-term adaptation of their lifestyle and life goals.

Might those with certain personality disorders respond differentially to different treatment regimes? Does the presence or absence of a personality disorder have implications for the prognosis or risk? How can this information be used for the treatment of CFS? Should a measurement of personality be included in a comprehensive assessment of CFS [30]? These are crucial questions that need to be addressed in future studies of personality and CFS. It is therefore important to consider our findings when conceptualizing a treatment regime for individuals with CFS. Our results indicate high correlations between personality traits and illness domains. Similarly high associations were found in the well and ISF groups. This means that there is indeed a strong association between personality and the experience of symptoms and feelings of impairment; however, this is not specific to ill people.

\section{Acknowledgments}

This research was supported in part by an appointment to the Research Participation Program at the CDC administered by the Oak Ridge Institute for Science and Education via an interagency agreement between the US Department of Energy and the CDC (U.M.N.). The findings and conclusions in this report are those of the authors and do not necessarily represent the views of the funding agency. The authors acknowledge the expertise of Rebecca Devlin and Marjorie Morrissey from Abt Associates in conducting the study. The authors would also like to thank Dr. Hao Tian (CDC) for the internal validation of the personality disorder data.

\section{Conflicts of Interest}

Drs. Nater, Jones, Lin, Maloney and Reeves report no competing interests. Dr. Heim has received, or currently receives, grant funding from the National Institute of Mental Health, the National Alliance for Research on Schizophrenia and Depression, the Anxiety Disorders Association of America, the Center for Behavioral Neuroscience/SNF, the CDC, Eli Lilly and Novartis. She has received consulting fees from Bristol Myers Squibb and CeNeRx. She has received a contractor salary from CDC. 


\section{References}

$\checkmark 1$ Reeves WC, Jones JF, Maloney E, Heim C, Hoaglin DC, Boneva RS, Morrissey M, Devlin R: Prevalence of chronic fatigue syndrome in metropolitan, urban, and rural Georgia. Popul Health Metr 2007;5:5.

$\checkmark 2$ Reyes M, Nisenbaum R, Hoaglin DC, Unger ER, Emmons C, Randall B, Stewart JA, Abbey S, Jones JF, Gantz N, Minden S, Reeves WC: Prevalence and incidence of chronic fatigue syndrome in Wichita, Kansas. Arch Intern Med 2003;163:1530-1536.

-3 Jason LA, Richman JA, Rademaker AW, Jordan KM, Plioplys AV, Taylor RR, McCready W, Huang CF, Plioplys S: A communitybased study of chronic fatigue syndrome. Arch Intern Med 1999;159:2129-2137.

$\checkmark 4$ Reynolds KJ, Vernon SD, Bouchery E, Reeves WC: The economic impact of chronic fatigue syndrome. Cost Eff Resour Alloc 2004;2:4.

$\checkmark 5$ Solomon L, Reeves WC: Factors influencing the diagnosis of chronic fatigue syndrome. Arch Intern Med 2004;164:2241-2245.

6 Bracken RM, Linnane DM, Brooks S: Plasma catecholamine and nephrine responses to brief intermittent maximal intensity exercise. Amino Acids 2009;36:209-217.

$\checkmark 7$ Ciccone DS, Busichio K, Vickroy M, Natelson $\mathrm{BH}$ : Psychiatric morbidity in the chronic fatigue syndrome: are patients with personality disorder more physically impaired? J Psychosom Res 2003;54:445-452.

8 Henderson M, Tannock C: Objective assessment of personality disorder in chronic fatigue syndrome. J Psychosom Res 2004;56: 251-254.

9 Pepper CM, Krupp LB, Friedberg F, Doscher C, Coyle PK: A comparison of neuropsychiatric characteristics in chronic fatigue syndrome, multiple sclerosis, and major depression. J Neuropsychiatry Clin Neurosci 1993; 5:200-205.

10 Johnson SK, DeLuca J, Natelson BH: Personality dimensions in the chronic fatigue syndrome: a comparison with multiple sclerosis and depression. J Psychiatr Res 1996;30:920 .
11 Costa PT Jr, McCrae RR: NEO PI-R professional manual. Odessa, Psychological Assessment Resources, Inc., 1992.

12 van Geelen SM, Sinnema G, Hermans HJ Kuis W: Personality and chronic fatigue syndrome: methodological and conceptual issues. Clin Psychol Rev 2007;27:885-903.

13 Appel S, Chapman J, Shoenfeld Y: Infection and vaccination in chronic fatigue syndrome: myth or reality? Autoimmunity 2007;40:48-53.

14 Magnusson AE, Nias DK, White PD: Is perfectionism associated with fatigue? J Psychosom Res 1996;41:377-383.

15 Buckley L, MacHale SM, Cavanagh JT, Sharpe M, Deary IJ, Lawrie SM: Personality dimensions in chronic fatigue syndrome and depression. J Psychosom Res 1999;46:395400.

16 Taillefer SS, Kirmayer LJ, Robbins JM, Lasry JC: Correlates of illness worry in chronic fatigue syndrome. J Psychosom Res 2003;54: 331-337.

17 Reeves WC, Wagner D, Nisenbaum R, Jones JF, Gurbaxani B, Solomon L, Papanicolaou DA, Unger ER, Vernon SD, Heim C: Chronic fatigue syndrome: a clinically empirical approach to its definition and study. BMC Med 2005;3:19.

-18 Fukuda K, Straus SE, Hickie I, Sharpe MC, Dobbins JG, Komaroff A: The chronic fatigue syndrome: a comprehensive approach to its definition and study. International Chronic Fatigue Syndrome Study Group. Ann Intern Med 1994;121:953-959.

19 Reeves WC, Lloyd A, Vernon SD, Klimas N, Jason LA, Bleijenberg G, Evengard B, White PD, Nisenbaum R, Unger ER: Identification of ambiguities in the 1994 chronic fatigue syndrome research case definition and recommendations for resolution. BMC Health Serv Res 2003;3:25.
20 Hyler SE: Personality Diagnostic Questionnaire-4+ (PDQ-4+). New York, New York State Psychiatric Institute, 1994.

21 American Psychiatric Association: Diagnostic and Statistical Manual of Mental Disorders, ed 4, text rev (DSM-IV-TR). Washington, American Psychiatric Association, 2000.

22 White C, Schweitzer R: The role of personality in the development and perpetuation of chronic fatigue syndrome. J Psychosom Res 2000;48:515-524.

$>23$ Kato K, Sullivan PF, Evengard B, Pedersen NL: Premorbid predictors of chronic fatigue. Arch Gen Psychiatry 2006;63:1267-1272.

24 Heim C, Wagner D, Maloney E, Papanicolaou DA, Solomon L, Jones JF, Unger ER, Reeves WC: Early adverse experience and risk for chronic fatigue syndrome: results from a population-based study. Arch Gen Psychiatry 2006;63:1258-1266.

25 Christodoulou C, Deluca J, Johnson SK, Lange G, Gaudino EA, Natelson BH: Examination of Cloninger's basic dimensions of personality in fatiguing illness: chronic fatigue syndrome and multiple sclerosis. J Psychosom Res 1999;47:597-607.

26 Harvey SB, Wadsworth M, Wessely S, Hotopf $\mathrm{M}$ : The relationship between prior psychiatric disorder and chronic fatigue: evidence from a national birth cohort study. Psychol Med 2008;38:933-940.

$\checkmark 27$ Bech P: Fifty years with the Hamilton scales for anxiety and depression: a tribute to Max Hamilton. Psychother Psychosom 2009;78: 202-211.

28 Fava GA, Sonino N: Psychosomatic assessment. Psychother Psychosom 2009;78:333341 .

29 Rimes KA, Chalder T: Treatments for chronic fatigue syndrome. Occup Med (Lond) 2005;55:32-39.

>30 Wise TN: Diagnostic criteria for psychosomatic research are necessary for DSM-V. Psychother Psychosom 2009;78:330-332. 